\title{
Effects of Altering Foot Position on Quadriceps Femoris Activation during Wall Squat Exercises
}

\author{
Yong-Jun Qiao, PT • Kyu-Ryeong Kim, PT, MS • Myoung-Kwon Kim, PT, PhD ${ }^{1 \dagger}$ \\ Department of Rehabilitation Sciences, Graduate School, Daegu University, \\ ${ }^{1}$ Department of Physical Therapy, College of Rehabilitation Sciences, Daegu University
}

Received: October 29, 2020 / Revised: November 23, 2020 / Accepted: December 21, 2020

(C) 2021 J Korean Soc Phys Med

\begin{abstract}
| Abstract |
PURPOSE: This study was conducted to identify the effects of altering foot position on quadriceps femoris including vastus medialis obliques (VMO), vastus lateralis (VL) and rectus femoris $(\mathrm{RF})$ activation during wall squat exercises.
\end{abstract}

METHODS: All subjects $(\mathrm{n}=15)$ were selected and randomly performed three kinds of wall squats: 1) GWS (General Wall Squat), 2) WSS1/4 (Wall Squat Short 1/4), and 3) WSS1/2 (Wall Squat Short 1/2). Each subject completed all three kinds of wall squatting exercises at three different times and recorded the muscle activity data of vastus medialis obliques, vastus lateralis and rectus femoris.

RESULTS: Compared with GWS exercise, VMO and RF muscle activity significantly increased under WSS1/2 exercise $(p<.05)$, while only RF muscle activity significantly increased under WSS1/4 exercise $(\mathrm{p}<.05)$.

CONCLUSION: The results of the present study indicate

$\dagger$ Corresponding Author : Myoung-Kwon Kim

skybird-98@hanmail.net, https://orcid.org/0000-0002-7251-6108 This is an Open Access article distributed under the terms of the Creative Commons Attribution Non-Commercial License (http://creativecommons.org/licenses/by-nc/3.0) which permits unrestricted non-commercial use, distribution, and reproduction in any medium, provided the original work is properly cited. that moving the foot toward the wall during wall squats has a positive effect on quadriceps activation. The exercise of wall squat short can not only be used as the lower limb muscle strengthening training for normal people, but also as the recovery training for patellofemoral pain syndrome patients in the rehabilitation stage. Besides, Anterior cruciate ligament patients can also try this exercise according to the advice of doctors and therapists.

Key Words: Vastus medialis obliques, Patellofemoral pain syndrome, Anterior cruciate ligament

\section{Introduction}

Quadriceps are essential for walking, standing, and using stairs in daily life. Muscle weakness may directly lead to impaired function, and people with weak muscles are much more likely to be disabled [1]. Intensive training of quadriceps femoris has many advantages. Strengthening the exercise of quadriceps femoris can reduce the pain and dysfunction of patients with knee osteoarthritis [2]. The strength and endurance of quadriceps femoris are very important to the normal function of knee joint, so the strengthening of quadriceps function after knee joint injury is an important part of rehabilitation [3], Not only in the 
rehabilitation of patellofemoral pain syndrome (PFPS) [4], but also in the recovery of anterior cruciate ligament (ACL) reconstruction surgery [5], the intensive training of quadriceps femoris plays a very important role.

The intensive training of quadriceps femoris is usually divided into open kinetic chain exercises and closed kinetic chain exercises [6]. Open kinetic chain exercises increase the muscle strength of the quadriceps femoris, thereby reducing the contact area between the patella and the femur, increasing shear stress and leading to excessive tension of the anterior cruciate ligament and nonfunctional muscles [7]. Therefore, open kinetic chain exercise training is not completely suitable for the recovery of muscle function during rehabilitation. Closed kinetic chain exercises have been shown to decrease anterior shear force and displacement of the tibia, as well as increase joint position sense and decrease patellofemoral contact stress [8]. So compared with open-kinetic chain exercise, closed-kinetic chain exercise is more effective for the activation of quadriceps femoris [9].

The squat exercise has been classified as a closed kinetic chain exercise [10]. Squatting is an integral part of daily functional exercises. Squatting can strengthen the muscles of the lower limbs and improve the ability to counteract the medial or lateral displacement of the knee [11]. Squatting exercise significantly improves the strength and endurance of the muscles of the lower extremities, especially the quadriceps femoris [12]. So squatting exercise is not only suitable for the patients with knee injuries as the best way to help restore the strength of the quadriceps muscle in overall activities of daily living [13], but also suitable for ordinary people as a daily exercise and health care project.

The quadriceps femoris consists of four individual muscles: three vastus muscles (vastus medialis, vastus intermedius, vastus lateralis) and the rectus femoris(RF). The imbalance between vastus medialis oblique(VMO) and vastus lateralis $(\mathrm{VL})$ can cause many problems. One of the factors affecting the balance of $\mathrm{VMO}$ and $\mathrm{VL}$ is the functional deviation. The fact that VL is activated earlier than VMO or VMO has a relatively small range of activation due to inhibition as a result of the pain or other answers may make the patella be drawn laterally [14]. So the position of the patella is mainly controlled by the dynamic action of the vastus medial oblique muscle [15]. That is to say, in order to enhance the dynamic stability of the patella, it is necessary to give priority to activating VMO muscles or to activate VMO muscles relatively more than VL muscles in an exercise. It was reported that the ratio of vastus medialis to vastus lateralis (VMO/VL) in patients with PFPS and normal people is 0.54 and 0.85 respectively [16]. The VMO/VL ratio of patients with PFPS was lower than that of normal people. Therefore, for PFPS patients, it is a good way to carry out effective exercise which can selectively give priority to strengthening VMO muscles in the early rehabilitation stage, while for normal people, this kind of exercise can better strengthen the stability of the knee and prevent the occurrence of knee-related diseases.

Common ways for changing squat exercises include changing standing width, foot placement angle, hip depth and extra load. In addition, gender and the ground environment when squatting may also be one of the influencing factors. However, the effects of these conditions on quadriceps femoris muscles have been studied by many people [17-19]. These studies were mainly aimed at general squatting positions such as partial squatting, half squatting, parallel squatting, full squatting and deep squatting [20].

There were relatively few studies on deformed squatting in other positions. Wall squatting was a common exercise used to train thigh muscles and buttocks. Physiotherapists can achieve the best results by using wall squatting, one-leg squatting and other similar exercises during the recovery of patellofemoral joint in PFPS patients [21]. Previous studies had studied the effects of four different ways of wall squatting on the activity of quadriceps femoris [22]. The result that wall squatting was more effective than 
regular squatting in strengthening muscle activity of VMO and VL has also been confirmed before [7]. Clinicians and trainers often believe that keep the knee in front of the toes during squatting exercises can increase patellofemoral force and stress, but there was no evidence to support the view, so Escamilla et al. [23] put forward the concept of "wall squat short" and studied the pressure data of patella and ligament under this exercise.

The effect of "wall squat short" on the activity of quadriceps femoris has not been studied, Wall squat short means to move the foot back in the normal wall squatting state. Therefore, in this study three different foot positions were selected for the experiment. So the purpose of this study was to investigate the effect of different foot position on the activity of quadriceps femoris muscle in wall squatting exercise, to determine which posture can strengthen quadriceps femoris better, and to recommend this method as a method of rehabilitation training after knee joint injury.

\section{Methods}

\section{Participants}

This study taken 15 healthy college students from Daegu University in South Korea as the research object. Before the beginning of the experiment, all the subjects understood the content of the study and signed the informed consent form. This study was approved by the Research Ethics Committee of Daegu University (IRB No.1040621-202007HR-001). The selection criteria were as follows: 1) professionals related to non-professional sports students and rehabilitation; 2) ability to squat for 15 to 30 minutes; and 3) there was no history of musculoskeletal or neurological diseases or disorders that had not affected the normal function of the hip, knee or ankle in the past 6 months. Exclusion criteria: 1) previous knee musculoskeletal injury or surgery; 2) knee and thigh pain; 3) open wound corresponding to the skin made it difficult to paste the electrode.

\section{Experimental procedures}

In this study, in order to compare the changes of quadriceps femoris before and after different wall squatting exercise. The general wall squat (GWS) position was to lean the body against the wall and then flexion the knees and hips to $90^{\circ}$. According to the position of the foot, we developed two different wall squat short (wall squat with feet closer to wall) postures. In the first wall squat short position, the foot position was the first quarter of the wall and foot position under the GWS position. In the second wall squat short position, the foot position was half the wall and foot position under the GWS position. These two wall squat short exercises can be respectively called to as WSS1/4 and WSS1/2 (Fig. 1).

Electrodes for electromyography (EMG) were attached

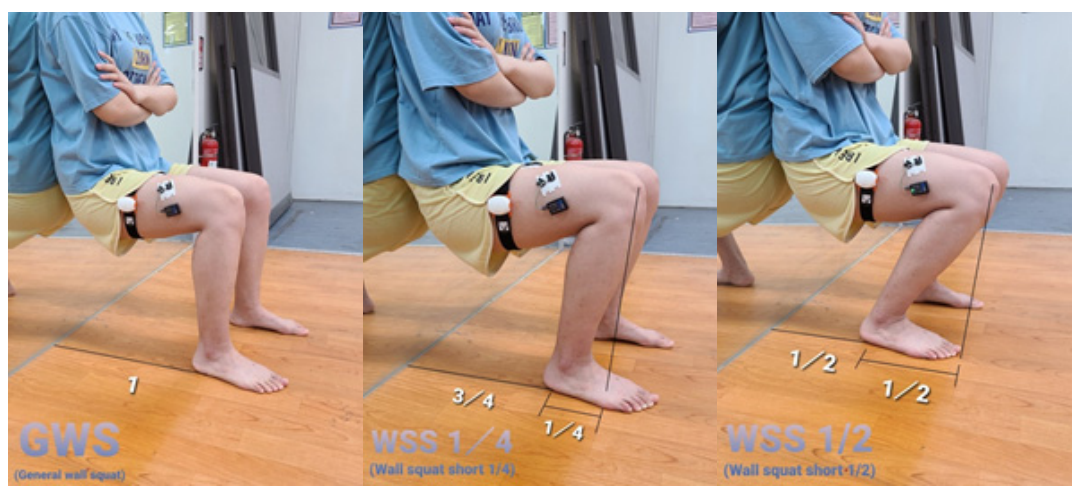

Fig. 1. Different wall squat exercises. 
to $\mathrm{VMO}$ muscle, $\mathrm{VL}$ muscle and $\mathrm{RF}$ muscle. When each subject was squatting, they first made sure that their legs are as wide as their shoulders, and stood with their backs directly against the wall, so that the hips and knees slided the thighs bend 90 degrees along the wall and the calves were parallel to the wall. In this GWS posture, the legs were roughly vertical, with the knees above the ankles. Then marked the position of the foot on the ground. The heel-to-wall distance for the WSS $1 / 2$ was the half of the distance for the GWS position, so the position of the foot under the posture of the WSS1/2 can be directly marked half of the position [23]. At the same time, marked the position of the foot under the WSS1/4 posture. During each exercise, an angle measuring instrument was used to control the subjects to maintain a 90 ' hip flexion. According to the tester's password, when the tester issues the "ready" password, the tester clung to the wall, then folds his arms in front of his chest, and when the tester said the "start" password, he or she officially entered the data testing process, which lasts for 5 seconds until the tester said "stop". The subject can have a rest. Tested three times at each angle, and after each measurement, in order to prevent repeated muscle fatigue, there was a 3-minute rest time after each measurement.

\section{Intervention}

In this study, all subjects tried to wear uniform shorts or loose clothing that fully exposed their thighs after signing the consent form. About 10 minutes of warm-up exercise needs to be completed before the experiment to prevent unnecessary injury during the formal experiment. The angle measuring instrument was bound to the thigh and determined that the standing position was $90^{\circ}$. The order of three squatting positions was selected according to the wishes of the subjects, and the subjects were given sufficient rest time after each action. After the subjects were familiar with the movements of the experiment, the surface electrode was used to record the EMG data of all the $\mathrm{VMO}$ muscle, the VL muscle and the RF muscle.

\section{Measurement}

\section{1) Surface Electromyography (EMG)}

In order to measure the muscle activity during wall squats, 16-channel radio-surface electrocardiogram (TeleMyoDTS, NoraxonIns, Az, USA) was required to collect myocardiogram data and process signals. The collected electrodynamic analogue signals were sent to Telemyo system DTS, converted to digital signals, and then filtered and processed other signals using Myoresearch XP 1.08 software (Noraxon Ins, AZ, USA). Sampling rate was set at $1,500 \mathrm{~Hz}$ for the measurement of activity. Frequency bandwidth was filtered using a band pass filter of 40 to $450 \mathrm{~Hz}$ and a notch filter of $60 \mathrm{~Hz}$ to quantify all the collected myocardial conduction signals, the collected signals for each muscle were treated as an effective mean (RMS), and the collected signals were treated as a percentage (\%) of the maximum numerical normal length contraction. To normalize the EMG data, maximal voluntary isometric contraction (MVIC) was performed for each muscle, and the EMG amplitude was recorded. All subjects first collected MVIC data from their right leg.

The subjects put on shorts or loose clothes, shaved the hair of the surface of the skin where the electrodes were attached, and wiped the skin with disposable medical alcohol test paper to ensure that the skin was clean and smooth. We positioned surface electrode pairs at an interelectrode distance of $2 \mathrm{~cm}$. The reference electrode was placed on the close position. For the VMO muscle, the electrodes placed at an oblique angle (55 degrees), 2 $\mathrm{cm}$ medially from the superior rim of the patella. For the VL muscle, two active electrodes were placed approximately 3 to $5 \mathrm{~cm}$ above the patella, on an oblique angle just lateral to midline. For the RF muscle, the two active electrodes were placed $2 \mathrm{~cm}$ apart, parallel to the 
muscle fibers [25]. Average value was used for data processing.

\section{2) Angle of hip flexion}

To visualize the movement of joints, the movements of subjects were accurately measured in real time using BPMpro Technology (Brown Spring, USA) and kept the angle of hip flexion $90^{\circ}$. To accurately measure the angle of knee flexion, placed the sensor vertically on the outside of the thigh, corresponding to the femur. Placed it as high as possible to prevent unnecessary interference from EMG measurement.

\section{Statistical Analysis}

The measured data were analyzed using SPSS (statistical package for the social sciences) version 24.0 for window software (SPSS Inc., Chicago). The Shapiro-Wilk test was used for the normality test. One-way repeated ANOVA was conducted to compare the statistical significance of changes in the muscle activity of the VMO, VL and RF on the different of wall squat exercises (GWS, WSS1/4

Table 1. General Characteristics of the Subjects $(n=15)$

\begin{tabular}{cc}
\hline Variables & Subjects \\
\hline Age (years) & $25.87 \pm 3.42^{\alpha}$ \\
Height $(\mathrm{cm})$ & $169.33 \pm 8.09$ \\
Weight $(\mathrm{kg})$ & $65.75 \pm 10.93$ \\
BMI & $22.82 \pm 2.80$ \\
\hline
\end{tabular}

${ }^{\alpha}$ Mean \pm SD, BMI: Body Mass Index and WSS1/2). A post-hoc analysis was performed using LSD test, and the level of significance was $p<.05$.

\section{Results}

A summary of general physical characteristics of the study subjects $(\mathrm{n}=15)$ was provided in Table 1 .

There was a significant difference in VMO according to three different wall squatting postures $(\mathrm{p}<.05)$ (Table 2$)$. When wall squatting in WSS1/2 position, the muscle activity of VMO was significantly higher than that in the normal squatting position $(\mathrm{p}<.05)$ (Table 2$)$. However, there was no significant difference in the change of VMO muscle activity between GWS posture and WSS1/4 posture, as well as between WSS1/4 posture and WSS1/2 posture $(\mathrm{p}>.05)$ (Table 2).

There was no significant difference in vastus lateralis according to three different wall squatting postures $(\mathrm{p}>$ .05) (Table 2).

There was a significant difference in rectus femoris according to three different wall squatting postures $(\mathrm{p}<$ .05) (Table 2). Compared with GWS, RF muscle activity was significantly increased in WSS1/4 and WSS1/2 postures $(p<.05)($ Table 2). But no significant difference was found between WSS1/4 posture and WSS1/2 posture $(\mathrm{p}>.05)$ (Table 2).

There was no significant difference in VMO/VL Ratio according to three different wall squatting postures $(\mathrm{p}>$ .05) (Table 3).

Table 2. EMG Activation of Quadriceps According to Three Different Wall Squatting Postures $(n=15)($ units: \%MVIC)

\begin{tabular}{ccccccc}
\hline Variable & GWS & WSS1/4 & WSS1/2 & F & p & Post-hoc \\
\hline VMO & $65.24 \pm 19.26^{\alpha}$ & $77.14 \pm 21.14$ & $89.26 \pm 25.00$ & 4.721 & $.014^{*}$ & WSS1/2 $>$ GWS \\
VL & $67.53 \pm 22.06^{\alpha}$ & $76.37 \pm 23.27$ & $83.39 \pm 25.46$ & 1.751 & .186 & - \\
RF & $47.37 \pm 21.46^{\alpha}$ & $68.33 \pm 26.85$ & $81.84 \pm 34.77$ & 5.987 & $.005^{*}$ & WSS $1 / 2>$ GWS, \\
WSS $1 / 4$
\end{tabular}

\footnotetext{
${ }^{\alpha}$ Mean \pm SD, VMO: Vastus medialis obliques, VL: Vastus lateralis, RF: Rectus femoris, GWS: General wall squat, WSS1/4: Wall squat short $1 / 4, \mathrm{WSS} 1 / 2$ : Wall squat short $1 / 2,{ }^{*} \mathrm{p}<.05$
} 
Table 3. EMG Activation of VMONL Ratio According to Three Different Wall Squatting Postures $(n=13)($ units: \%MVIC)

\begin{tabular}{ccccccc}
\hline Variable & GWS & WSS1/4 & WSS1/2 & F & p & Post-hoc \\
\hline VMO/VL & $.88 \pm .14^{\alpha}$ & $.92 \pm .17$ & $.97 \pm .16$ & 1.364 & .268 & \\
\hline
\end{tabular}

${ }^{\alpha}$ Mean \pm SD, VMO: Vastus medialis obliques, VL: Vastus lateralis, RF: Rectus femoris, GWS: General wall squat, WSS1/4: Wall squat short $1 / 4, \mathrm{WSS} 1 / 2$ : Wall squat short $1 / 2, * \mathrm{p}<.05$

\section{Discussion}

In this study, in order to understand the changes of three lower limb muscles and VMO/VL ratio in three different wall squatting postures, the muscle activity data of $\mathrm{VMO}$, VL and RF were measured by \%MVIC method. It can be seen from the results of this study that the EMG activity of $\mathrm{VMO}$ and $\mathrm{RF}$ muscles was significantly different between the GWS and WSS1/2 postures and the EMG activity of RF muscles was significantly different between the GWS and WSS1/4 posture. In addition, VMO/VL ratio did not change significantly under any of the three wall squats.

During wall squatting, if the position remained motionless, the whole body received four external forces, namely, the force of the wall mirror, the gravity of the body, the upward force of the ground at the feet and the friction of the ground [26]. We can choose the contact point between the foot and the ground as the fulcrum, because it was in a motionless state, so the resultant moment should be 0 , that was, the sum of the four moments mentioned above was 0. Compared with the GWS movement, when the WSS1/4 and WSS1/2 movement, because the position of the foot was closer to the wall, so the position of the hip joint would move down, and the gravity arm would also be shortened, so the wall would have more support to the back. The main muscle corresponding to the wall support force for the back is RF muscle, so the RF muscle activity was significantly changed during different wall squatting exercises.

When keeping the movement still, the knee joint stretching torque had done positive work in order to overcome the influence of gravity. The concentric contraction of the vastus lateral muscle almost maximized the recruitment of the $\mathrm{VMO}$, while RF also had a high level of muscle activity [27]. When the center of gravity was transferred to the front of the base plane, the muscle activity of the RF was reduced, and the extension torque was also reduced, that was, it would lead to the decrease of the muscle activity of the knee extensor [28]. On the contrary, during the WSS1/2 exercise, the center of gravity line was transferred backward, which would lead to an increase in the activity of the knee extensor, and the quadriceps femoris, as the main knee extensor, would show an increase in activity. From the data, we can also see that the VMO, VL and RF had a numerical trend of increasing as the foot position was further back.

One of the main causes of PFPS is the muscle imbalance of the lower extremities. The abnormal relationship between VMO and VL muscle activation patterns may interfere with the dynamics of the patellofemoral joint $[2,29]$. The VMO was the only muscle that provided a medial force [30], and many exercise treatments for patients with PFPS also emphasized the importance of VMO because of its medial force on the patella [4,31-33]. According to the experimental results, there was a significant difference in the change of VMO. Compared with GWS exercise, all the three muscles of WSS1/2 exercise had an increasing trend, but the increase of VMO was larger than that of $\mathrm{VL}$, so for the priority strengthening of VMO muscle, the exercise of WSS1/2 was more advantageous. Because many studies also put forward the significance of the VMO/VL ratio, we also made a statistical analysis of the VMO/VL data, although there was no statistical difference in the 
results, but on the whole, with the foot position moving backward, the $\mathrm{VMO} / \mathrm{VL}$ ratio had an increasing trend. This also indirectly proved that under the condition of wall squatting, with the backward movement of the foot position, the activation of lower limb muscles, especially VMO muscle and RF muscle, would significantly increase, which can not only exercise the quadriceps femoris more effectively, but also meet the training needs of PFPS patients in the later stage of rehabilitation.

Escamilla et al. [23] compared the effects of wall squat long and wall squat short on cruciate ligament force and patellofemoral joint force and stress. Patellofemoral force and stress were greater during the wall squat short compared with the wall squat long at $90^{\circ} \mathrm{knee}$ angle, this may be due to the use of greater quadriceps strength. Although the strength of the quadriceps used in the wall squat short exercise is greater than the quadriceps muscle used in the wall squat long exercise, but the strength of the posterior cruciate ligament (PCL), that was, the back shear force, was smaller. In the stage of wall squatting, the cruciate ligament forces were provided by PCL and would not generate ACL forces. So for patients with ACL reconstruction, perhaps a shorter distance between the foot and the wall for wall squatting can not only get better quadriceps exercise, but also less damage to ACL.

The subjects of this experiment were mainly healthy college students, which lead to the fact that the results of this experiment may not be fully applicable to patients with knee injury. Although WSS1/2 exercise had a significant effect on quadriceps muscles, it also increased the pressure on the patella, which was why it is recommended that patients with knee injury squat at an angle of knee flexion less than $50^{\circ}$ during early rehabilitation training [10]. Compared with GWS exercise, in the WSS1/4 exercise, although only RF muscle activity has a statistically significant increase, but in terms of numerical value, all three muscles have an increasing trend. So for physiotherapists and doctors, it was more important to choose a better exercise mode according to specific rehabilitation goals and training programs. As for whether there was a better position within the distance between the foot and the corner of the wall that can effectively strengthen the quadriceps femoris and does not affect the patellar stress, further research is needed.

\section{Conclusion}

In this study, 15 subjects were measured for quadriceps muscle activity during wall squats in different foot positions to determine which posture could better strengthen quadriceps or be suitable for rehabilitation training after knee injuries. Just from the point of view of quadriceps muscle strengthening, moving the foot back during wall squatting produces greater $\mathrm{VMO}$ and $\mathrm{RF}$ muscle activation, resulting in muscle strengthening. As a rehabilitation training method for knee injuries, patients with PFPS were more effective when wall squatting with their feet further back. This experiment also provides some theoretical results for clinical rehabilitation.

\section{References}

[1] O'Reilly SC, Jones A, Muir KR, et al. Quadriceps weakness in knee osteoarthritis: the effect on pain and disability. Annals of the Rheumatic Diseases. 1998;57(10):588-94.

[2] Jun I, Lee Y, Shin C. The Effects of Isometric Extension at Different Knee Angles on Vastus Medialis Electromyographic Activity in Patients with Knee Joint Osteoarthritis. J Phys Ther Sci. 2012;24(9):855-7.

[3] Hart JM, Pietrosimone B, Hertel J, et al. Quadriceps activation following knee injuries: a systematic review. J Athl Train. 2010;45(1):87-97.

[4] Singh GK, Srivastava S. Preferential strengthening of VMO muscle during selected biomechanical rehabilitative exercises of automotive workers with patellofemoral pain syndrome. Work. 2018;60(1):135-41. 
[5] Palmieri-Smith RM, Thomas AC, Wojtys EM. Maximizing quadriceps strength after ACL reconstruction. Clin Sports Med. 2008;27(3):405-24.

[6] Yuen CHN, Lam CPY, Tong KCT, et al. Investigation the EMG activities of lower limb muscles when doing squatting exercise in water and on land. Int J Environ Res Public Health. 2019;16(22):4562.

[7] Jun Cheol L, Min AG. Effect of general squat and wall squat exercises on the muscle activity of the vastus medialis oblique and vastus lateralis. Journal of International Academy of Physical Therapy Research. 2017;8(3): 1248-54.

[8] Blanpied PR. Changes in muscle activation during wall slides and squat-machine exercise. J Sport Rehabil. 1999;8(2):123-34.

[9] Irish SE, Millward AJ, Wride J, et al. The effect of closed-kinetic chain exercises and open-kinetic chain exercise on the muscle activity of vastus medialis oblique and vastus lateralis. J Strength Cond Res. 2010;24(5): 1256-62.

[10] Escamilla RF. Knee biomechanics of the dynamic squat exercise. Med Sci Sports Exerc. 2001;33(1):127-41.

[11] Lorenzetti S, Ostermann M, Zeidler F, et al. How to squat? Effects of various stance widths, foot placement angles and level of experience on knee, hip and trunk motion and loading. BMC Sports Sci Med Rehabil. 2018;10(1):14

[12] Lee JY, Lee DY. Effect of different speeds and ground environment of squat exercises on lower limb muscle activation and balance ability. Technol Health Care. 2018;26(4):593-603.

[13] Jeong HW, Chon SC. Comparison of the electromyographic changes in the vastus medialis oblique and vastus lateralis muscles according to the knee joint angle during squat exercise using a gym ball. J Korean Soc Phys Ther. 2016;23(4):9-15.

[14] Hung YJ, Gross MT. Effect of foot position on electromyographic activity of the vastus medialis oblique and vastus lateralis during lower-extremity weight-bearing activities. J Orthop Sports Phys Ther. 1999;29(2):93-105.

[15] Earl JE, Schmitz RJ, Arnold BL. Activation of the VMO and $\mathrm{VL}$ during dynamic mini-squat exercises with and without isometric hip adduction. J Electromyogr Kinesiol. 2001;11(6):381-6

[16] Kim HH, Song CH. Effects of knee and foot position on EMG activity and ratio of the vastus medialis oblique and vastus lateralis during squat exercise. Journal of muscle and joint health. 2010;17(2):142-150.

[17] Ricky A, Carol C, Eli C. EMG analysis of the vastus medialis/vastus lateralis muscles utilizing the unloaded narrow-and wide-stance squats. J Sport Rehabil. 1998; 7(4):236-47.

[18] Murray N, Cipriani D, O'Rand D, et al. Effects of foot position during squatting on the quadriceps femoris: an electromyographic study. Int J Exerc Sci. 2013;6(2): 114-25.

[19] Jaberzadeh S, Yeo D, Zoghi M. The effect of altering knee position and squat depth on VMO : VL EMG ratio during squat exercises. Physiother Res Int. 2016;21(3): 164-73.

[20] Kitamura T, Kido A, Ishida Y, et al. Muscle activity pattern with a shifted center of pressure during the squat exercise. J Sports Sci Med. 2019;18(2):248-52.

[21] Escamilla RF, Zheng N, Macleod TD, et al. Patellofemoral joint force and stress during the wall squat and one-leg squat. Med Sci Sports Exerc. 2009;41(4):879-88.

[22] Kim BJ. Comparison of quadriceps femoris muscle activations during wall slide squats. Journal of the Korean Society of Physical Medicine. 2012;7:541-50.

[23] Escamilla RF, Zheng N, Imamura R, et al. Cruciate ligament force during the wall squat and the one-leg squat. Med Sci Sports Exerc. 2009;41(2):408-17.

[24] Schaub PA, Worrell TW. EMG activity of six muscles and $\mathrm{VMO}: \mathrm{VL}$ ratio determination during a maximal squat exercise. J Sport Rehabil. 1995;4(3):195-202.

[25] Criswell E. Cram's introduction to surface electromyography 
(2nd ed). Sudbury. Jones and Bartlett Publishers. 2010.

[26] Biscarini A, Contemori S, Dieni CV, et al. Joint torques and tibiofemoral joint reaction force in the bodyweight "Wall Squat" therapeutic exercise. Applied Sciences. 2020;10(9):3019.

[27] Robertson DG, Wilson JM, St Pierre TA. Lower extremity muscle functions during full squats. J Appl Biomech. 2008;24(4):333-9.

[28] Lee TS, Song MY, Kwon YJ. Activation of back and lower limb muscles during squat exercises with different trunk flexion. J Phys Ther Sci. 2016;28(12):3407-10.

[29] Thomeé R, Augustsson J, Karlsson J. Patellofemoral pain syndrome: a review of current issues. Sports Med. 1999;28(4):245-62.
[30] Dixit S, DiFiori JP, Burton M, et al. Management of patellofemoral pain syndrome. Am Fam Physician. 2007; 75(2):194-202.

[31] Mc CJ. The management of chondromalacia patellae: a long term solution. Aust J Physiother. 1986;32(4): 215-23.

[32] Grabiner MD, Koh TJ, Miller GF. Fatigue rates of vastus medialis oblique and vastus lateralis during static and dynamic knee extension. J Orthop Res. 1991;9(3):391-7.

[33] Barak Y, Ayalon M, Dvir Z. Spectral EMG changes in vastus medialis muscle following short range of motion isokinetic training. J Electromyogr Kinesiol. 2006;16(5): 403-12. 